\title{
Aerospace panels fixtureless inspection methods with restraining force requirements; a technology review
}

\author{
Gad N. Abenhaim ${ }^{\mathrm{a}, *}$, Antoine S. Tahan ${ }^{\mathrm{b}}$, Alain Desrochers ${ }^{\mathrm{a}}$, Jean-Francois Lalonde ${ }^{\mathrm{c}}$ \\ ${ }^{a}$ Université de Sherbrooke, Sherbrooke, Canada \\ ${ }^{b}$ École de technologie supérieure, Montreal, Canada \\ ${ }^{c}$ Bombardier Aerospace, Montreal, Canada
}

\begin{abstract}
Aerospace panels are commonly restrained on complex inspection fixture jigs during the measurement process. Forces used to restrain the parts are also monitored as mandated by thier functional requirements. Given the difficulties in measuring these types of parts, this paper reviews the available fixtureless inspection methods with a focus on the challenges of their implementation, and their aptitude to be used to estimate the profile and the necessary restraining forces of an aerospace panel. To perform this investigation, finite element analysis is used to predict the constrained shape of four (4) simulated free-state aerospace panels, with two different type of boundary conditions, in five scenarios. From those analyses, the importance and limits of current finite element boundary setting methods embedded in fixtureless inspection methods for nonrigid parts are highlighted.
\end{abstract}

Keywords: Dimensional metrology, Fixtureless Inspection, Finite Element Method, Boundary conditions, Nonrigid parts

\section{Introduction}

Aerospace panels present particular challenges during their geometrical and dimensioning inspection due to their complex shape, nonrigid (or compliant) behavior, and specific technical requirements (e.g., profile and waviness). For example, a typical skin panel of an aircraft as shown in Fig. 1 can be lightly warped at free-state condition, making the value of its profile measurement exceed its requirements, thus making the quality of the part unacceptable. This same panel may nevertheless be assembled and conformed to the airframe therefore making its profile measurement fall within the specified requirement. This example highlights the delicacy of inspection, if insufficient fixing constraints are applied, to ensure, first, the measurement

\footnotetext{
*. Corresponding author. Tel.: +1-819-821-7111; fax: +1-819-821-7163.

Email address: gad-noriel .abenhaim@usherbrooke.ca (Gad N. Abenhaim)
} 


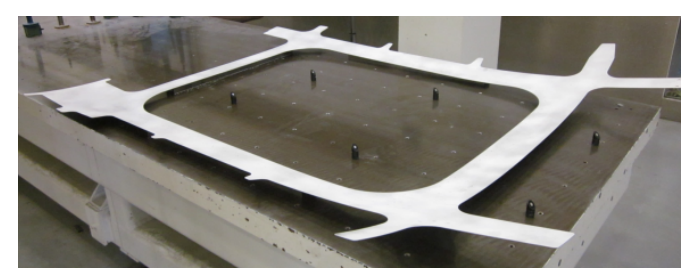

Figure 1: Example of an aerospace panel in a free-state. (source: Bombardier aerospace)

setup properly represents the assembly functionality of the part and, second, the measurement's repeatability and reproducibility.

For such nonrigid parts, the American Society of Mechanical Engineers (ASME Y14.5 [1]) and International Organization for Standardization (ISO 10579 [2]) standards' principle is that the restrained conditions must be specifically stated on the drawing, on an associated document or in a CAD product definition data set. This principle can give way to a vast amount of restraining condition specification methods. Nevertheless, as proposed by Abenhaim et al. [3], restrained condition specification methods can be classified into five methods contingent if the part is to be restrained:

1. On a shaped fixture

2. On a hyperstatic datum reference frame

3. By movable datum targets

4. On a basic dimension

5. By a force

For large nonrigid parts, such as aerospace panels, their functional requirements also mandate a limit to the constraints imposed during the assembly. Given these challenges, aerospace panels are commonly restrained on complex inspection fixture jigs during the measurement process as shown in Fig. 1. The restraining method for these types of parts limits to some given values the forces which may restrain the part during inspection. Typically, a note such as "A load of X N/m can be used to achieve tolerance." is indicated next to the associated requirement. Commonly, there is no requirement on the spatial location of the applied loads for an aerospace panel application.

The complexity of the inspection fixtures as well as the difficulties and time consuming process of evaluating the restraining forces are draining much-needed resources from the metrology departments. Therefore, recent trends in inspection methods for such non-rigid parts are focused on decreasing the inspection cost by removing the need for the use of dedicated inspection fixtures. An actively investigated approach by researchers in the field is referred to as the simulated displacements method [3]. This approach to the fixtureless inspection of nonrigid parts relies on a numerical approach to virtually compare the shape of the measured part in free-state condition with its nominal CAD model. Accordingly, this paper reviews these methods with a focus on the challenges of their implementation, and their aptitude to be used to estimate the profile and the restraining forces that are commonly monitored during the assembly of aerospace parts. To this end, the aerospace panel shown in Fig. 1 is used to highlight the limits of the underlying principal of those methods to estimate partsâẮz profile deviation and the aforementioned restraining forces. 
Before any substantive discussion can transpire from this paper, it is necessary to take a moment to explain the way in which it will unfold. Section 1 has introduced the challenges involved in specifying and inspecting the geometric and dimensional requirements of nonrigid parts. Having established these challenges, Sec. 2 provides an overview of existing research into the fixtureless inspection methods for nonrigid parts to emphasize their underlying principal. Section 3 studies the aerospace panel shown in Fig. 2 in different scenarios to highlight the state of art of fixtureless method for their use in the aerospace industry. Finally, Sec. 5 describes the directions of future research.

\section{Fixtureless inspection}

To allow the evaluation of the surface profile of a part, the measured surface points must be compared to their nominal positions defined by their design model. Two types of measurement methods are used for free-form surface inspection: contact measurements traditionally using a touch probe mounted on a coordinate measuring machine (CMM), and noncontact measurements - such as laser and optical scanning or photogrammetry. The point cloud generated from either method must be compared to the CAD model in order to evaluate the deviation of the part with respect to its tolerances. When designed, the CAD model exists in the design coordinate system (DCS), while the measurement method generates a point cloud in the measurement coordinate system (MCS). When using an inspection fixture, unifying these two coordinate systems is straightforward. Alternatively, the process of merging these two coordinate systems is referred to as registration, localization, or alignment. Mathematically, registration refers to finding an optimal transformation matrix between the DCS and the MCS. This registration process removes the need for expensive fixtures and reduces the inspection setup time. This is true only when dealing with rigid parts, since the purpose of inspection fixtures when dealing with nonrigid parts is twofold - aligning the part in a reference frame and maintaining the part in a desired shape during inspection. Thus far, registration methods have been widely developed for non-contact inspection approaches for rigid parts $[4,5]$.

Registration methods for nonrigid parts need to fulfill the two aforementioned purposes of the inspection fixture. The challenge to overcome by these methods is to compensate for the shape changes of the nonrigid part when it is not fixed to its design shape. The aforementioned simulated displacements methods [6-16] rely on a numerical approach to virtually compare the shape of the measured part in free-state and its nominal CAD model. The comparison is done by imposing displacements on either the part's free-state or its nominal CAD to force it into taking the shape of the other.

Preserving the industry practice of comparing the restrained manufactured part with its nominal CAD model, methods such as $[8-12,15,16]$ propose the use of a numerical fixture instead of a hard fixture to constrain the part to its functional shape. These methods essentially consist in building a finite element (FE) model of the scanned manufactured part in a free-state and imposing the boundary conditions on the FE model using the known position of the nominal fixating points on the CAD model. The resulting FE model simulates the scanned part fixed onto its inspection fixture. The following step consists in comparing this virtual fixed part with the nominal CAD model, which allows for the evaluation of the profile deviations. By doing so, those methods require the pre-processing of the point cloud of the part so as to convert it into a tessellated surface and then into a suitable mesh for a FE analysis. A material calibration procedure may also be necessary to refine the FE model [9]. Notwithstanding the endeavor of 


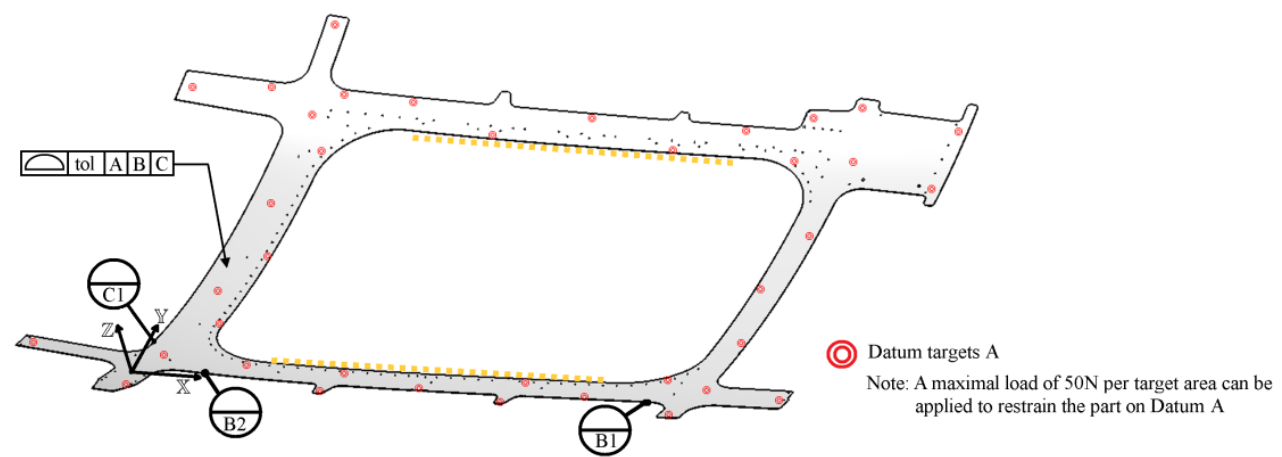

Figure 2: Nominal model with: red dots representing 38 datum targets of $\mathbf{A}, 2$ datum targets $\mathbf{B}$, 1 datum target $\mathbf{C}$. Dashed lines represent the boundaries used to simulate the part's free-state shapes.

building an appropriate FE model from a points cloud, imposing the relevant boundary conditions on the FE model is a major factor contributing to the reliability of the FE analysis results [17].

Given the large size and nonrigid behavior of common aerospace panels, a high amount of restraining points, used to constrain the part to its nominal position, must be represented by boundary conditions in a FE model. This particular requirement augments the importance of the boundary conditions compared to simple nonrigid shapes necessitating 3-4 restraining points. Therefore, the next section studies the limits of the classic use of finite element model into fixtureless inspection method for nonrigid parts. Specifically, the study investigates the limits of the boundary conditions setting method in finite element software for use in fixtureless inspection methods, in the hypothetical situation where: $(i)$ the scanned part is pre-processed to a highly accurate finite element mesh, (ii) the properties of material are known, (iii) thickness of part is uniform and known, and (iv) the position of the fixing point on scanned part are known. It should be noted that finding the restraining points positions on the part at free-state is a challenging task since nonrigid part do not take their nominal shape at free-state. Additionally, finding the restraining points' positions of an aerospace panel is difficult as they do not have easily identified positions such as would be for a fixation hole.

\section{Case study}

The case study herein is an aerospace aluminum panel of approximately $1425 \mathrm{~mm}$ [56 in] width by $1730 \mathrm{~mm}$ [68 in] of length with an area of $0,592 \mathrm{~m}^{2}$ [917.6 $\left.\mathrm{in}^{2}\right]$, as shown in Fig. 1 . The panel has a thickness of $2.54 \mathrm{~mm}$ [0.1 in]. The part reference frame, illustrated in Fig. 2, is constituted by the thirty eight (38) datum targets depicting primary datum $\mathbf{A}$, two datum targets representing the secondary datum $\mathbf{B}$ and the one tertiary datum target $\mathbf{C}$. In a free-state, the part's shape varies significantly from its nominal shape as shown in Fig. 1 and 5. An over-constrained fixture such as shown in Fig. 3 is therefore necessary to adequately evaluate the part's profile.

To perform this investigation, finite element analysis is used to predict the constrained shape of four (4) simulated free-state aerospace panels, with two different type of boundary conditions, in five scenarios, as detailed in the next subsections. 


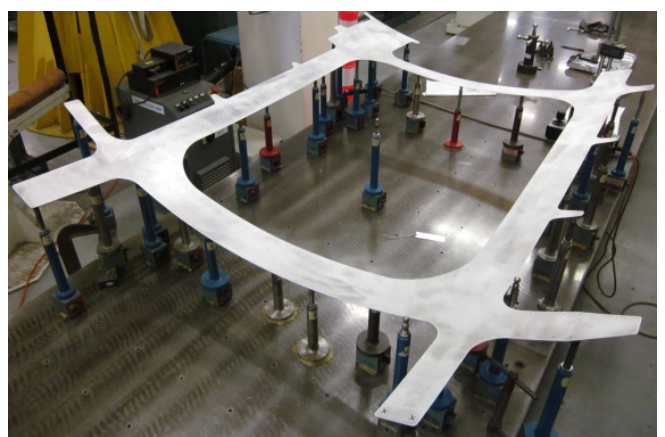

Figure 3: Example of an aerospace panel constrained on its inspection fixture before the measurement process. (source: Bombardier aerospace)

\subsection{Freestate shape simulation}

To produce the aforementioned hypothetical situation where: $(i)$ the scanned part is preprocessed to a highly accurate finite element mesh, (ii) the properties of material are known, (iii) thickness of part is uniform and known, and (iv) the position of the fixing point on scanned part are known, four (4) scanned manufactured parts at free-state are simulated. Starting with the CAD model of the part, the four parts are simulated using the following steps schematized in Fig.4:

1. A FE mesh with $4 \mathrm{~mm}$ spacing (37 752 nodes) of the nominal model is generated using the software ANSYS ${ }^{\circledR}$ and is referred herein as FreeState\#0.

2. A FE analysis using the mesh FreeState\#O is performed to generate a plausible deformed shape of the part. This analysis simulates the part at free-state mounted on two beams represented by dashed line on Fig. 2 . The resulting deformed shape is referred as FreeState\#1. The displacements on FreeState\#0 generating FreeState\#1 are shown in Fig. 5 .

3. A measurement noise which follows a normal distribution centered at 0 with a standard deviation of $0.05 \mathrm{~mm}$ is introduced on the FreeState\#1, thus creating FreeState\#2.

4. An additional outliers measurement noise which follows a normal distribution centered at 0 with a standard deviation of $0.30 \mathrm{~mm}$ is introduced on $2.5 \%$ of the nodes of FreeState\#2, resulting in FreeState\#3.

\subsection{Boundary conditions}

Having established the simulated free-state parts, the next step consists in setting the boundary conditions of the finite element model. Boundary conditions must represent the most accurately possible the effect of the surrounding environment on the model for the situation being simulated. In practical term, boundary conditions must be representative of how the part is restrained on its physical fixture. Commonly during the inspection process, aerospace panel are first positioned on their reference frame at free-state. Then, the part is forced on each datum targets $\mathbf{A}$ on the physical fixture (one by one), and maintained during the rest on the process. Therefore two boundary configurations are studied. 


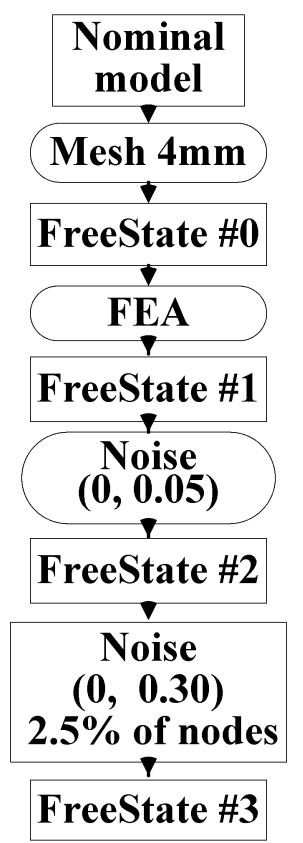

Figure 4: Process simulating the four free-state parts.

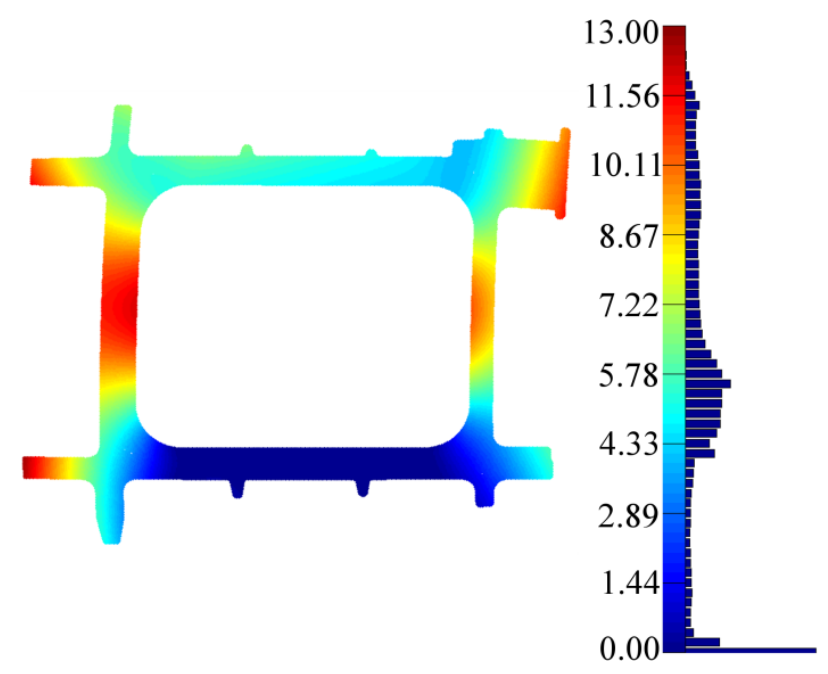

Figure 5: Displacements in millimeters on FreeState\#0 generating FreeState\#1. 


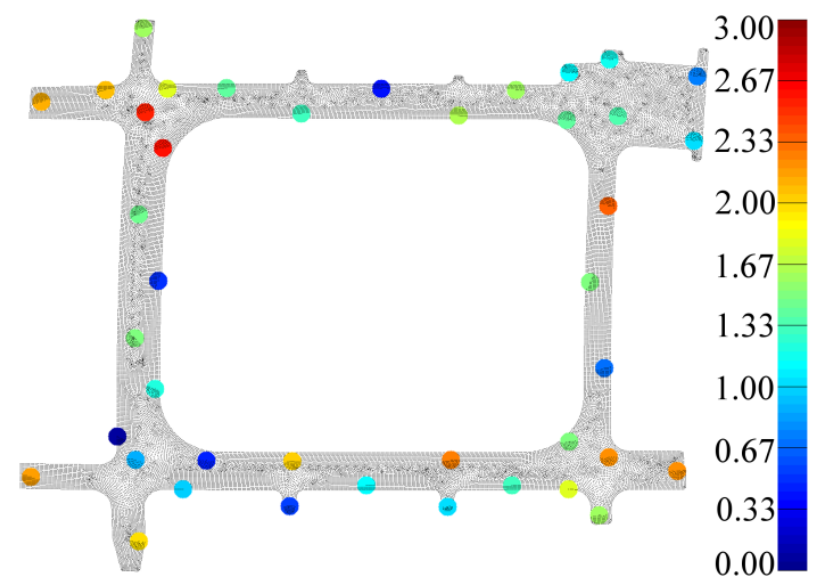

Figure 6: Distance in millimeters between the nodes $\mathcal{B}^{0}$ of the nominal mesh Freeestate\#0 and the nominal position of the 41 datum targets $\mathbf{B}$.

The first configuration, Config I, consists in imposing: $(i)$ a displacement in the $\mathcal{Z}$ direction to all the nodes corresponding to datum targets $\mathbf{A}$, (ii) a displacement in the $\mathcal{Y}$ direction to all the nodes corresponding to the datum targets $\mathbf{B}$, (iii) a displacement in the $\mathcal{X}$ direction to the nodes corresponding to the datum target $\mathbf{C}$, and (iv) the gravity load.

The second configuration, Config. II, consists in imposing: (i) a displacement in the $\mathcal{X} \& \mathcal{Y} \& \mathcal{Z}$ directions to all the nodes corresponding to the datum targets $\mathbf{A}$, (ii) a displacement in the $\mathcal{Y}$ direction to all the nodes corresponding to the datum targets $\mathbf{B}$, (iii) a displacement in the $\mathcal{X}$ direction to the nodes corresponding to the datum target $\mathbf{C}$, and (iv) the gravity load.

\subsection{Scenarios}

Taken into account the precedent simulated free-state parts and the boundary conditions configurations, this section details five (5) scenarios in which FE analysis is used to predict the constrained shape of a free-state part. Specifically, each scenario describes the input, boundary conditions and output of the FE analysis is used to predict the constrained shape of the part as detailed in Table 1. For each scenario, the constrained degrees of freedom of the boundaries are as per each boundary configuration presented in the previous subsection.

Let $\mathcal{B}$ be a set of the nominal position of the forty one (41) datum targets and $\mathcal{B}^{0}$ be the set of nodes of the mesh Freeestate\#0 closest to $\mathcal{B}$. Figure 6 illustrates the distances between $\mathcal{B}^{0}$ and $\mathcal{B}$. Additionally, let $\mathcal{B}^{1}$ be the position of the set of nodes $\mathcal{B}^{0}$ of the mesh Freeestate\# $; \mathcal{B}^{2}$ be the position of the set of nodes $\mathcal{B}^{0}$ of the mesh Freeestate\#2; $\mathcal{B}^{3}$ be the position of the set of nodes $\mathcal{B}^{0}$ of the mesh Freeestate\#3.

The first scenario consists in maintaining the Freestate\#0 FE mesh by imposing null displacements as boundary conditions on nodes $\mathcal{B}^{0}$ while applying the gravity load. This allows the evaluation of the initial errors of the model and to verify that sufficient restraining datum targets have been imposed. The resulting deformed mesh is referred as Pridected\#1.

Scenario II constrains the Freestate\#1 FE mesh by imposing that nodes $\mathcal{B}^{1}$ be at the position of the nodes $\mathcal{B}^{0}$, as well as the gravity load. This simulates the situation where the hypothetical highly accurate FE mesh of the scanned part at free-state is deformed to match its nominal model. The resulting deformed mesh is referred as Pridected\#2. 
Scenario III constrains the Freestate\#2 FE mesh by imposing that nodes $\mathcal{B}^{2}$ be at the position of the nodes $\mathcal{B}^{0}$, as well as the gravity load. This simulates the situation where the hypothetical highly accurate FE mesh of the scanned part at free-state includes a Gaussian noise of $N(0,0.05) \mathrm{mm}$ due to the measurement process. The resulting deformed mesh is referred as Pridected\#3.

Scenario IV constrains the Freestate\#3 FE model by imposing that nodes $\mathcal{B}^{3}$ be at the position of the nodes $\mathcal{B}^{0}$, as well as the gravity load. This simulates the situation where the hypothetical highly accurate FE mesh of the scanned part at free-state includes a noise of $N(0,0.05) \mathrm{mm}$ and some outliers Gaussian noise of $N(0,0.30) \mathrm{mm}$ due to the measurement processing. The resulting deformed mesh is referred as Pridected\#4.

Finally, scenario $V$ constrains the Freestate\#3 FE mesh by imposing that nodes $\mathcal{B}^{3}$ be at the position of the nominal datum targets positions $\mathcal{B}$, as well as the gravity load. This simulates the situation where there is not necessarily a node on the hypothetical highly accurate FE mesh at the exact positions of the nominal datum targets positions $\mathcal{B}$. The resulting deformed mesh is referred as Pridected\#5.

Table 1: Summary of the different scenarios with their respective predicted shape outputs. *The gravity load is applied on all the models.

\begin{tabular}{llll}
\hline \hline Scenarios & FEA Input & Boundary conditions* & Output \\
\hline$\# 1$ & FreeState\#0 & $\mathcal{B}^{0} \Rightarrow \mathcal{B}^{0}$ & Predicted\#1 \\
$\# 2$ & FreeState\#1 & $\mathcal{B}^{1} \Rightarrow \mathcal{B}^{0}$ & Predicted\#2 \\
$\# 3$ & FreeState\#2 & $\mathcal{B}^{2} \Rightarrow \mathcal{B}^{0}$ & Predicted\#3 \\
$\# 4$ & FreeState\#3 & $\mathcal{B}^{3} \Rightarrow \mathcal{B}^{0}$ & Predicted\#4 \\
$\# 5$ & FreeState\#3 & $\mathcal{B}^{3} \Rightarrow \mathcal{B}$ & Predicted\#5 \\
\hline \hline
\end{tabular}

\section{Results and Discussion}

To assess the limit of the classic use of finite element model into fixtureless inspection method, this section compares each predicted shape described in the previous section with the part nominal CAD model. Additionally, the reaction forces of each predicted shape are investigated. A summary of the results is presented in Table 2.

\subsection{Shape evaluation}

Given that the correspondence between each node of the predicted mesh and the nominal mesh are known, the distance between each corresponding node is used to evaluate the predicted shape model error.

Firstly, from the initial shape errors of Predicted\#1 shape shown in Fig. 7(a)(b), it can be inferred that both boundary conditions configurations are acceptable, and that there are sufficient restraining datum targets. Though, the boundary configuration II introduces lower shape errors.

Figure 8 depicts the mean and maximal shape errors per predicted shape for each boundary configurations. From this figure, it can be concluded that even given the hypothetical highly accurate FE mesh of the scanned part, as the scenario complexity augments and tends to resemble a real world application, the shape errors of the predictive shape augments. 


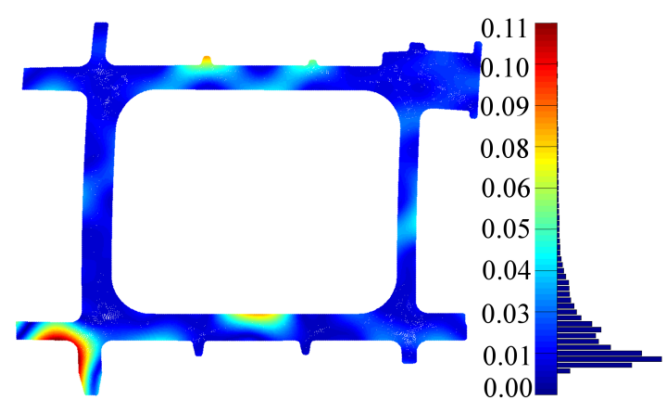

(a)

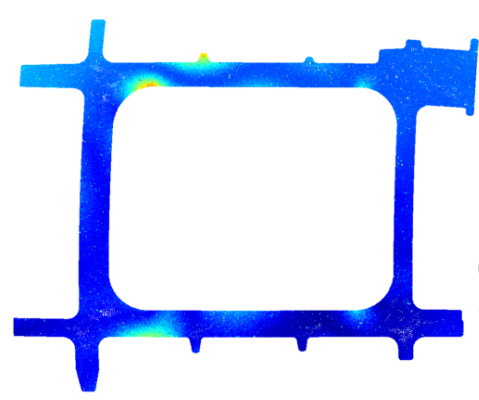

(c)

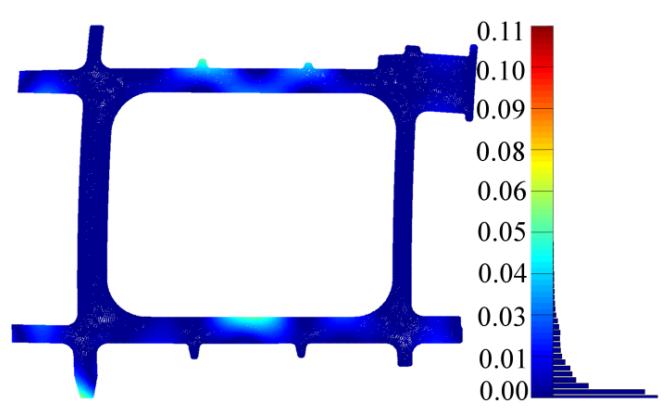

(b)

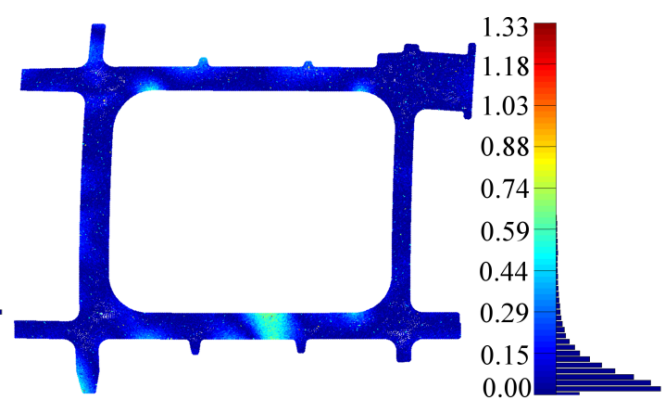

(d)

Figure 7: Shape errors in millimeters for:

(a) Predicted\#1 - Configuration I (b) Predicted\#1 - Configuration II

(c) Predicted\#4 - Configuration I (d) Predicted\#4 - Configuration II

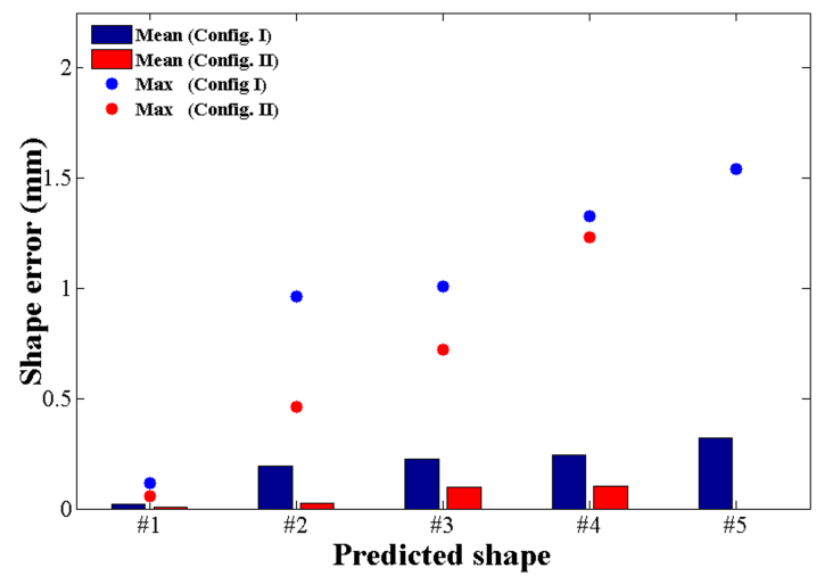

Figure 8: Mean and maximum values of the shape errors in millimeters of each predicted shapes per boundary configuration. No reliable Predicted\#5 using the boundary conditions configuration II was obtained. 
Though Fig. 7 shows that the boundary configuration II introduces lower shape errors for the predicted shapes \#1 to \#4, no viable solutions were found for Predicted\#5. Table 2 shows that the estimated stress values for Predicted\#5 are extremely high, suggesting that the estimated deformation is not in the elastic domain. That is, given a small uncertainties in the boundary positions due to the mesh density (i.e., lack of node at the exact location of the datum targets) of the model renders the boundary configuration II invalid even though this configuration introduces less shape errors for the scenarios resulting in Predicted\#1 to 4.

Finally, despite the improbable hypothetical situation simulated since real world applications have more uncertainties, Fig. 9 shows that the uncertainties in the boundary positions due to the model mesh density (see Fig. 6) is sufficient to give way to a unacceptably high shape errors of the predicted shapes for an aerospace panel application.

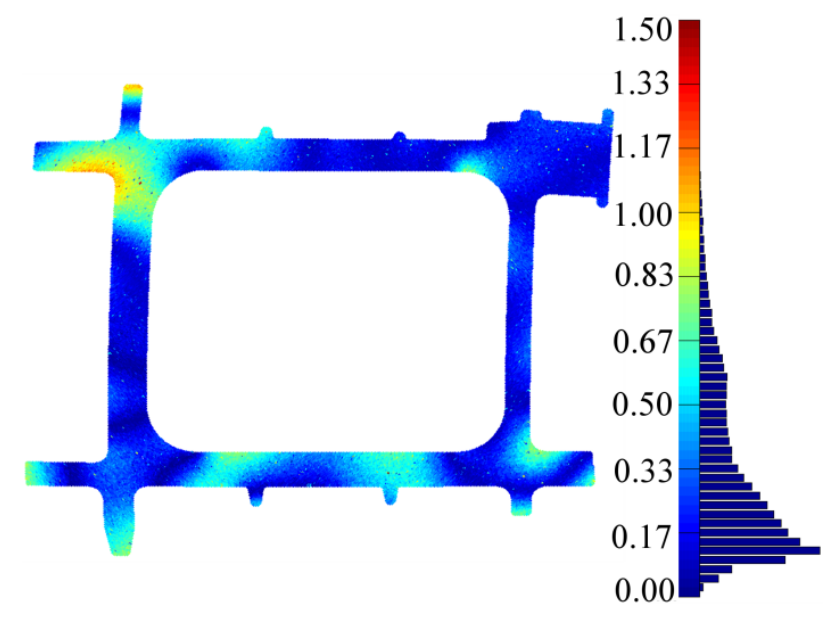

Figure 9: Shape errors in millimeters of Predicted\#5- Configuration I.

Table 2: Summary for each predicted shape for the boundary conditions as per configuration I/ configuration II.

\begin{tabular}{ccclcc}
\hline \hline $\begin{array}{c}\text { Predicted } \\
\text { Shape }\end{array}$ & \multicolumn{2}{c}{ Shape Error $(\mathrm{mm})$} & \multicolumn{2}{c}{ Force $(N)$} & \multicolumn{1}{c}{ Mean } \\
Max & \multicolumn{1}{c}{ Mean } & \multicolumn{1}{c}{ Max } & Max \\
\hline$\# 1$ & $0.021 / 0.006$ & $0.114 / 0.058$ & $0.48 / 2.65$ & $1.49 / 8.62$ & $1.48 / 0.98$ \\
$\# 2$ & $0.194 / 0.027$ & $0.963 / 0.460$ & $1.23 / 17.78$ & $5.87 / 72.23$ & $18.19 / 22.37$ \\
$\# 3$ & $0.225 / 0.096$ & $1.011 / 0.724$ & $1.31 / 153.16$ & $5.62 / 763.61$ & $19.56 / 35.17$ \\
$\# 4$ & $0.244 / 0.100$ & $1.327 / 1.230$ & $1.31 / 153.78$ & $5.62 / 763.37$ & $19.64 / 34.81$ \\
$\# 5$ & $0.321 /$ na & $1.544 /$ na & $1.56 /$ na & $8.86 /$ na & $18.35 / 3.09 \mathrm{e}+003$ \\
\hline \hline
\end{tabular}

\subsection{Force estimation}

As mentioned previously, aerospace panel's specifications mandate that the force necessary to constrain the part to its fixture does not exceed a maximum force. Therefore, the estimated reaction forces at the boundary conditions are examined. Figure 10 presents the mean and maximal reaction forces at the boundary nodes per predicted shape for each boundary 
configuration. From this figure, it can be concluded that contrary to the shape errors, the boundary configuration I is more appropriate with respect to the force evaluation. Reason is that even for small uncertainty in the position boundary conditions, the boundary configuration II will tend to locally elongate or contract the part, thus resulting in high reaction forces at the boundary nodes.

Though Fig. 10 shows that as the scenario complexity augments and tends to resemble a real world application, the boundaries conditions values gives way to higher reaction forces. The forces variations for configuration II suggest that this configuration model is acceptable with respect to the forces estimations.

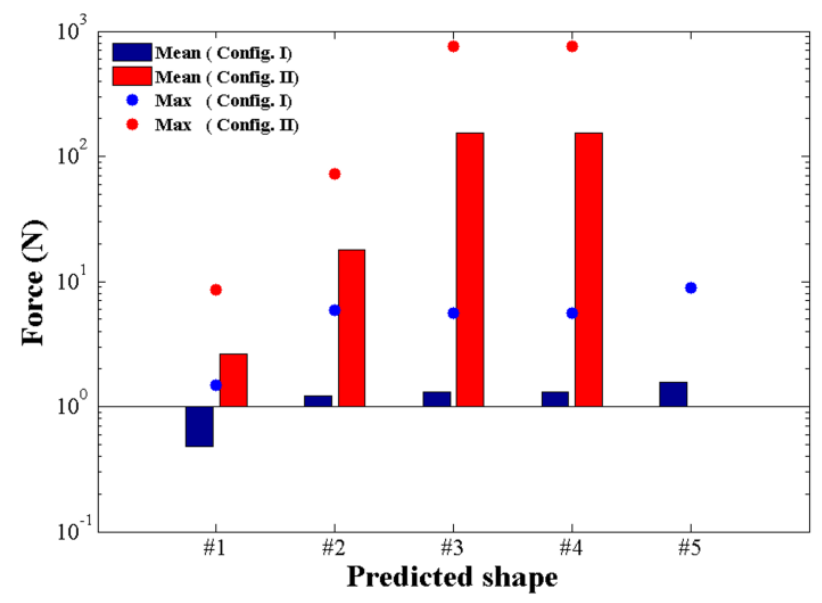

Figure 10: Mean and maximum values of the reaction forces in Newtons at the boundary nodes of each predicted shapes per boundary configuration. No reliable Predicted\#5 with the boundary conditions configuration II was obtained.

\section{Summary and Conclusion}

By investigating the limits of the boundary conditions setting methods in FE software in fixtureless inspection methods, this study highlighted the challenges and aptitude of FE-based simulated displacements methods to estimate nonrigid parts' profile and restraining forces. Through the use of a hypothetical situation where most uncertainties associated with a real world applications are removed, this study finding demonstrate that the boundary conditions configurations and values are major factors contributing to the reliability of the FE analysis results predicting the shape of nonrigid parts mounted onto their fixture. Given two possible boundary conditions configurations used to simulate an aerospace panel maintained on its inspection fixture, this investigation reveals that a compromised between the accuracy of the predicted shapes and the estimated reaction forces must be made when choosing a proper boundary conditions configuration. More importantly, despite the improbable hypothetical situation simulated, this study shows that uncertainties in the boundary positions due to the model mesh density (i.e., lack of node at the exact location of the datum targets, see Fig. 6) are sufficient to give way to a unacceptably high shape errors of the predicted shape for an aerospace panel application. Therefore, it can be concluded that at the present state of art of FE boundary setting 
methods, FE-based simulated displacements methods still need key developments before they can be applied to for the inspection of real world aerospace panels.

\section{Acknowledgments}

The authors would like to thank the National Sciences and Engineering Research Council, Université de Sherbrooke, École de Technologie Supérieure and Bombardier aerospace for their support and financial contribution. The authors would also like to express their gratitude to Ali Aidibe of École de Technologie Supérieure, as well as Dominic Moreau, Andre Leduc and Brent Jackle of Bombardier aerospace for their help and advices.

\section{References}

1. ASME-Y14.5, . Dimensioning and Tolerancing. New York, NY: American Society of Mechanical Engineers; 2009.

2. ISO-10579, . Geometrical Product Specifications (GPS) - Dimensioning and tolerancing - Non-rigid parts. International Organization for Standardization (ISO); 2010.

3. Abenhaim, G.N., Desrochers, A., Tahan, A.S.. Nonrigid parts' specification and inspection methods: notions, challenges, and recent advancements. The International Journal of Advanced Manufacturing Technology 2012; 63(5-8):741-752.

4. Li, Y., Gu, P.. Free-form surface inspection techniques state of the art review. Computer Aided Design 2004; 36(13):1395-1417.

5. Savio, E., De Chiffre, L., Schmitt, R.. Metrology of freeform shaped parts. CIRP Annals - Manufacturing Technology 2007;56(2):810-835.

6. Abenhaim, G.N., Tahan, A.S., Desrochers, A., Maranzana, R.. A novel approach for the inspection of flexible parts without the use of special fixtures. Journal of Manufacturing Science and Engineering, Transactions of the ASME 2011;133(1):011009 (pages 11). doi:|bibinfo $\{$ doi $\}\{10.1115 / 1.4003335\}$.

7. Aidibe, A., Tahan, A.S., Abenhaim, G.N.. Distinguishing profile deviations from a part's deformation using the maximum normed residual test. WSEAS Transactions on Applied and Theoretical Mechanics 2012;7(1):18-28.

8. Blaedel, K., Swift, D., Claudet, A., Kasper, E., Patterson, S.. Metrology of non-rigid objects. Tech. Rep. UCRL-ID-146957; Lawrence Livermore National Lab.; 2002.

9. Gentilini, I., Shimada, K.. Predicting and evaluating the post-assembly shape of thin-walled components via 3D laser digitization and FEA simulation of the assembly process. Computer-Aided Design 2011;43(3):316-328.

10. Jaramillo, A.E., Boulanger, P., Prieto, F.. On-line 3-D inspection of deformable parts using FEM trained radial basis functions. In: IEEE 12th International Conference on Computer Vision Workshops (ICCV Workshops). 2009, p. $1733-1739$.

11. Jaramillo, A., Boulanger, P., Prieto, F.. Fixtureless inspection of deformable parts using partial captures. International Journal of Precision Engineering and Manufacturing 2013;14(1):77-83.

12. Radvar-Esfahlan, H., Tahan, S.A.. Nonrigid geometric metrology using generalized numerical inspection fixtures. Precision Engineering 2012;36(1):1-9.

13. Weckenmann, A., Gabbia, A.. Testing formed sheet metal parts using fringe projection and evaluation by virtual distortion compensation. In: Fringe 2005. 2006, p. 539-546.

14. Weckenmann, A., Weickmann, J.. Optical inspection of formed sheet metal parts applying fringe projection systems and virtual fixation. Metrology and Scientific Instrumentation 2006;13(4):321-334.

15. Weckenmann, A., Weickmann, J., Petrovic, N.. Shortening of inspection processes by Virtual Reverse Deformation. In: 4th International Conference and Exhibition on Design and Production of Machines and Dies/Molds. 2007,

16. Weickmann, J.. Assistenzsystem für die prüfung von blechbauteilen mit mehrkomponentenstreifenprojektionssystemen unter anwendung der "Virtuellen Rückdeformation". Thesis; Universität ErlangenNürnberg; 2010.

17. Lemeš, S.. Validation of numerical simulations by digital scanning of $3 D$ sheet metal objects. Ph.D. thesis; University of Ljubljana; Ljubljana; 2010 